\title{
Validation of chromogenic in situ hybridization for detection of EGFR copy number amplification in nonsmall cell lung carcinoma
}

\author{
Lynette M Sholl ${ }^{1}$, A John Iafrate ${ }^{2}$, Yi-Ping Chou ${ }^{3}$, Ming-Tsang Wu ${ }^{4}$, Yih-Gang Goan ${ }^{3}$, Li Su ${ }^{5}$, \\ Yen-Tsung Huang ${ }^{5}$, David C Christiani ${ }^{5,6}$ and Lucian R Chirieac ${ }^{1}$
}

${ }^{1}$ Department of Pathology, Brigham and Women's Hospital, Harvard Medical School, Boston, MA, USA; ${ }^{2}$ Department of Pathology, Massachusetts General Hospital, Harvard Medical School, Boston, MA, USA; ${ }^{3}$ Department of Chest Surgery, Kaohsiung Veteran General Hospital, Kaohsiung, Taiwan; ${ }^{4}$ Department of Family Medicine, Graduate Institute of Occupational Safety and Health, Kaohsiung Medical University Hospital, Kaohsiung, Taiwan; ${ }^{5}$ Department of Environmental Health, Harvard School of Public Health, Boston, MA, USA and ${ }^{6}$ Pulmonary and Critical Care Unit, Department of Medicine, Massachusetts General Hospital, Harvard Medical School, Boston, MA, USA

\begin{abstract}
Epidermal growth factor receptor (EGFR) gene copy number correlates with response to tyrosine kinase inhibitors in patients with nonsmall cell lung carcinoma. Fluorescence in situ hybridization (FISH), a standard methodology to detect EGFR copy number abnormalities in nonsmall cell lung carcinoma, is limited by instrumentation and cost. Chromogenic in situ hybridization (CISH) is an emerging alternative detection technique using light microscopy, but its utility in assessing EGFR copy number in lung cancer is not established. To address the utility of $\mathrm{CISH}$, we studied paraffin-embedded nonsmall cell lung carcinoma specimens from 77 Taiwanese nonsmoking women treated by surgery alone. We recorded the number of signals per tumor cell nucleus, correlated EGFR copy number by CISH with FISH results, and used receiver operating characteristics to identify cut-off points for the CISH results. Tumors were classified as adenocarcinoma $(n=28)$, mixed adenocarcinoma with bronchioloalveolar features $(n=25)$, bronchioloalveolar carcinoma $(n=2)$, squamous cell carcinoma $(n=15)$, and adenosquamous carcinoma $(n=7)$. By FISH, $29 \%$ of cases had no amplification, $18 \%$ had low polysomy, $35 \%$ had high polysomy, and $12 \%$ had gene amplification. EGFR copy number detected by $\mathrm{CISH}$ highly correlated with FISH (Spearman $r=0.81, P<0.0001$ ). We determined the optimal EGFR CISH cut-off points that discriminate between no amplification and low polysomy (2.8 signals, $P=0.09) ;$ no amplification plus low polysomy and high polysomy plus gene amplification (4.5 signals, $P<0.0001$ ); and high polysomy and gene amplification (7.1 signals, $\boldsymbol{P}=0.0003$ ). $\mathrm{CISH}$ is an alternative assay to FISH in determining EGFR copy number status that may contribute to stratification of patients with nonsmall cell lung carcinoma for clinical trials and identify a subset of patients that should be treated with tyrosine kinase inhibitors. Modern Pathology (2007) 20, 1028-1035; doi:10.1038/modpathol.3800946; published online 3 August 2007
\end{abstract}

Keywords: EGFR; lung cancer; chromogenic in situ hybridization

Hyperactivation of epidermal growth factor receptor (EGFR), a member of the ErbB family of receptor tyrosine kinases, has been implicated in the pathogenesis of numerous carcinomas, most notably nonsmall cell lung carcinoma. ${ }^{1}$ Recognized mechanisms of EGFR gain-of-function in nonsmall cell lung carcinoma include somatic activating mutations

Correspondence: Dr LR Chirieac, MD, Department of Pathology, Brigham and Women's Hospital, 75 Francis Street, Boston, MA 02115, USA.

E-mail: lchirieac@partners.org

Received 21 April 2007; revised 30 June 2007; accepted 3 July 2007; published online 3 August 2007 in the exons encoding the tyrosine kinase domain ${ }^{2}$ and EGFR gene amplification. ${ }^{3}$ The EGFR-specific tyrosine kinase inhibitors erlotinib (Tarceva; OSI Pharmaceuticals, Genentech) and gefitinib (Iressa; AstraZeneca) have provided clinical responses of varying degree in a subset of patients with nonsmall cell lung carcinoma who are predominantly nonsmoking women of East Asian descent. ${ }^{4-6}$

The majority of patients with nonsmall cell lung carcinoma who respond to tyrosine kinase inhibitors harbor a somatic EGFR mutation, ${ }^{7}$ although $10-20 \%$ have no identifiable mutation. ${ }^{1}$ A proportion of patients without EGFR mutations and at least a partial clinical response to tyrosine kinase 
inhibitors have been shown to have an increased EGFR gene copy number in their tumor cells. ${ }^{8,9}$ The coexistence of EGFR gene amplification and mutation is of unclear clinical significance; however, some data suggest that gene amplification confers increased sensitivity to tyrosine kinase inhibitors in tumors with EGFR mutations. ${ }^{8,10}$ Particularly in US and European populations, where EGFR mutations are less common than in Asian populations, ${ }^{11,12}$ the status of the EGFR gene copy number appears important in predicting overall survival following tyrosine kinase inhibitor therapy in patients with advanced nonsmall cell lung carcinoma. ${ }^{13}$

Although fluorescence in situ hybridization (FISH) is an established technique for assessing EGFR gene amplification in nonsmall cell lung carcinoma, it is expensive, time consuming, and requires a special protocol, materials, and fluorescent microscopy. In contrast, chromogenic in situ hybridization (CISH) utilizes a peroxidase reaction to detect the locus of interest and can be performed in the clinical immunohistochemistry laboratory and interpreted by standard light microscopy. The reliability of CISH as a technique for detecting gene amplification has been established for HER2 in breast cancer specimens. ${ }^{14-16}$ However, there are no published reports that examine the utility of CISH in detecting EGFR copy number in lung cancer specimens. In the present study, we evaluated the utility of CISH in detecting EGFR copy number by comparing EGFR CISH results with FISH results in a cohort of 77 nonsmoking Taiwanese women with nonsmall cell lung carcinoma, and used discriminant analysis to identify CISH cut-off points that differentiate between different EGFR copy number categories.

\section{Materials and methods}

Specimens were obtained from 77 patients with nonsmall cell lung carcinoma treated by surgery alone at the Kaohsiung Veteran General Hospital, Taiwan from 1999-2004. All patients were women and had a nonsmoking history. The study was approved by the institutional review boards of the Kaohsiung Veteran General Hospital and Kaohsiung Medical University and all participants gave written informed consent. Hematoxylin and eosin-stained slides from methanol-fixed, paraffin-embedded tissue sections were reviewed and classified according to the World Health Organization criteria, ${ }^{17,18}$ and staged according to Edition 6 of the American Joint Committee on Cancer. ${ }^{19}$ A representative area containing predominantly tumor was selected for FISH and CISH analyses on consecutive tissue sections.

\section{FISH}

FISH was carried out on paraffin-embedded tissue sections. Briefly, sections were baked at $65^{\circ} \mathrm{C}$ for $2 \mathrm{~h}$ to fix the tissue to the slide, then the tissue was deparaffinized with three changes of fresh xylene for 30,10 , and $10 \mathrm{~min}$. Xylene was removed with two rounds of $100 \%$ ethanol treatment for 2 min each. The slides were then placed in boiling $100 \mathrm{mM}$ Tris/50 mM EDTA for $10 \mathrm{~min}$. Slides were washed with $2 \times$ SSC for $5 \mathrm{~min}$, followed by two rounds of digestion with Digest-All 3 (Zymed-Invitrogen, South San Francisco, CA, USA) for $35 \mathrm{~min}$ each. The tissue was then dehydrated in 70,95 , and $100 \%$ ethanol for 2 min each.

DNA probes to EGFR (BAC no. CTD-2113A18) and to 7q (BAC no. CTB-1013N12) were directly labeled via Nick Translation with SpectrumGreen and SpectrumOrange fluorophores, respectively (Vysis, Downers Grove, IL, USA). Probes were applied to the designated area of the slide and slides were coverslipped and sealed with rubber cement. Slides were placed in a humidified oven and the probes were denatured at $80^{\circ} \mathrm{C}$ for $5 \mathrm{~min}$, followed by hybridization at $37^{\circ} \mathrm{C}$ overnight (HYBrite, Abbott). After hybridization, slides were washed twice in $50 \%$ formamide $/ 2 \times$ SSC and twice in $2 \times$ SSC for 5 min each at $45^{\circ} \mathrm{C}$. Slides were counterstained with DAPI (Abbott) and coverslipped.

The signals were evaluated with a fluorescent microscope (Olympus BX60 Fluorescence Microscope/Camera, Center Valley, PA, USA) containing SpectrumOrange, SpectrumGreen, and DAPI filters. Two pathologists (LMS and AJI) simultaneously evaluated at least 50 cells for each case, with no knowledge of the CISH status.

The EGFR copy number was classified by the number of copies per cell according to previously published criteria $^{8,21}$ as no amplification, low genomic gain or low polysomy $(<4$ copies of $E G F R$ in $>40 \%$ of cells), high polysomy ( $\geq 4$ copies of EGFR in $>40 \%$ of cells), and gene amplification (homogenously staining regions with $\geq 15$ copies in $\geq 10 \%$ of cells or a gene/chromosome ratio per cell of $\geq 2$, Figure 1).

\section{CISH}

For CISH, paraffin-embedded tissue sections were deparaffinized in two changes of xylene for $5 \mathrm{~min}$ each; xylene was removed in three washes of ethanol for 3 min each $(100,100$, and $95 \%)$ and the slides were washed in distilled running water for $5 \mathrm{~min}$. The slides were placed in heated $\left(>90^{\circ} \mathrm{C}\right)$ CISH Pretreatment Buffer (Invitrogen) and microwaved on high power for $30 \mathrm{~min}$, then rinsed in distilled water for $5 \mathrm{~min}$ at room temperature. The tissue was digested for $10 \mathrm{~min}$ with pepsin digestion solution (Invitrogen) at room temperature, washed twice in distilled water for 5 min each, dehydrated in 90 , 95, and $100 \%$ alcohol for 2 min each, then dried in a $37^{\circ} \mathrm{C}$ oven.

Five to seven microliters of EGFR Amplification Probe (Zymed-Invitrogen) were applied to the 

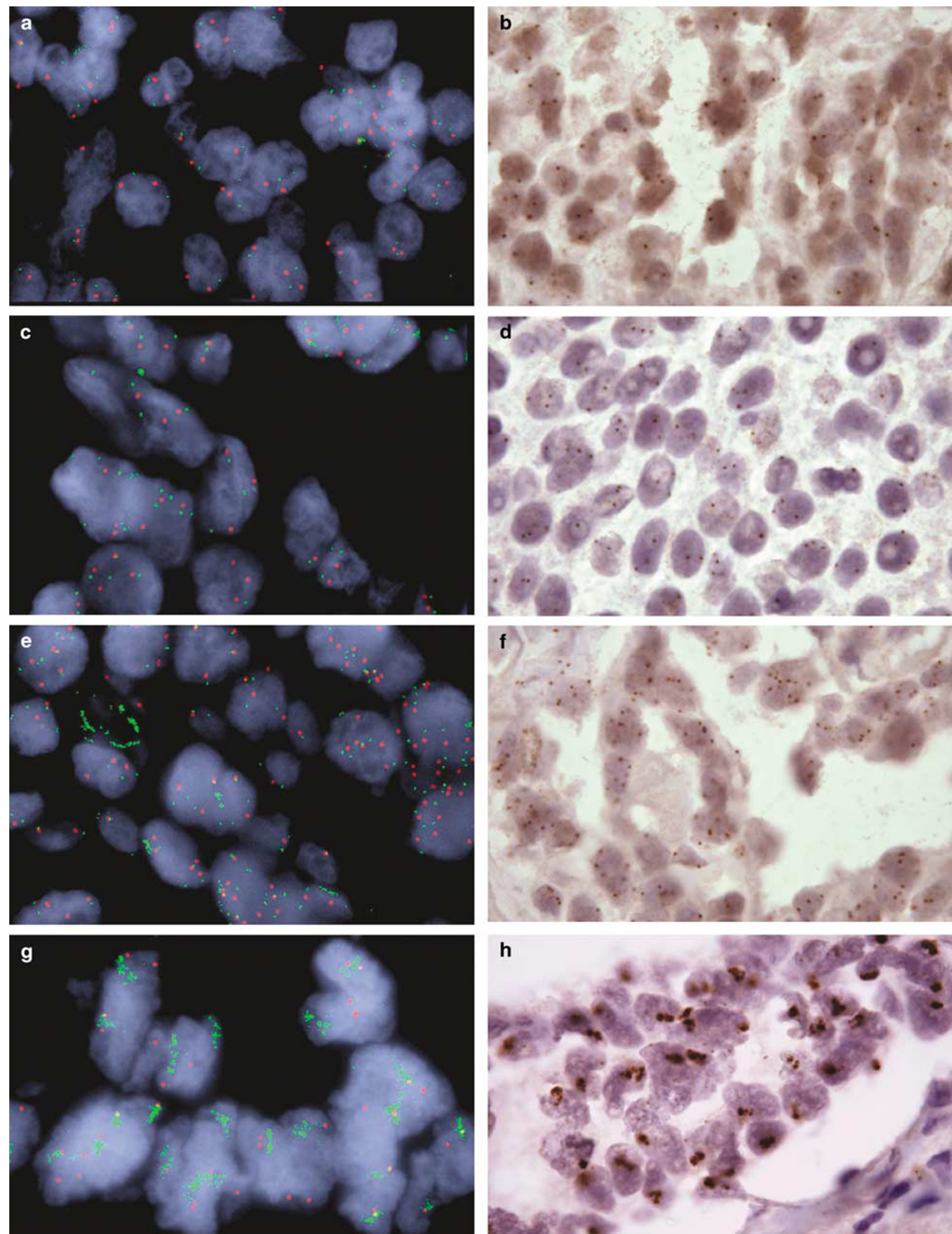

Figure 1 FISH with an EGFR probe (green) and 7q control probe (red) in nonsmall cell lung carcinoma sections demonstrating (a) no amplification, (c) low polysomy, (e) high polysomy, and (g) gene amplification. The corresponding slides treated by CISH with the EGFR probe are shown in (b), (d), (f), and (h). 
designated area and a coverslip was applied and sealed with rubber cement. The slide was dried at $37^{\circ} \mathrm{C}$, followed by probe denaturation at $95^{\circ} \mathrm{C}$ for $5 \mathrm{~min}$ and hybridization at $37^{\circ} \mathrm{C}$ overnight in a Thermobrite oven (MarketLab, Caledonia, MI, USA). The slides were washed in $0.5 \%$ SSC for $5 \mathrm{~min}$ at room temperature, followed by $0.5 \%$ SSC for $5 \mathrm{~min}$ at $75^{\circ} \mathrm{C}$, and water for $5 \mathrm{~min}$ at room temperature.

For immunodetection, slides were placed in 3\% hydrogen peroxide in absolute methanol for $10 \mathrm{~min}$ then washed in phosphate-buffered saline with $0.025 \%$ Tween 20 (PBST) three times for $2 \mathrm{~min}$ each. Slides were incubated with nonspecific blocking solution (Zymed-Invitrogen) for $10 \mathrm{~min}$ at room temperature, then incubated with mouse antidigoxigenin antibody for $30 \mathrm{~min}$ at room temperature, washed in PBST twice for $2 \mathrm{~min}$ each, and incubated with horseradish peroxidase-conjugated goat anti-mouse antibody for $15 \mathrm{~min}$, and finally washed again with PBST twice for $2 \mathrm{~min}$ each. The slides were then incubated with $\mathrm{DAB}$ chromagen (Zymed-Invitrogen) for $30 \mathrm{~min}$ at room temperature and washed in distilled water twice for $2 \mathrm{~min}$ each. Slides were counterstained with hematoxylin.

The CISH-prepared slides were examined at $\times 400$ by bright field microscopy. Two pathologists (LMS and LRC) examined the cases concomitantly and agreed on CISH scores; cases for which there was disagreement were further reviewed and a consensus was established. Both examiners were blinded to the FISH results. As in prior studies, ${ }^{20}$ 200 tumor nuclei were examined in each case (Figure 1). In the cases where the number of signals per tumor nucleus varied between tumor cells, the range of signals counted was recorded for each case.

\section{Statistical Analysis}

A geometric average was calculated from the range of CISH scores to represent the point estimate for each case. The CISH point estimate was weighted based on accuracy according to the equation: weight $=$ least value in range/greatest value in range. The EGFR copy number as determined by CISH was compared with EGFR copy number determined by FISH for each case. CISH scores were correlated with FISH scores using nonparametric analysis (Spearman's correlation). Receiver operating characteristics (ROC) curves were derived from a logistic regression model using CISH signal point estimates to discriminate between categories. The cut-off points with the highest sensitivity and specificity for estimating EGFR copy number by CISH were derived from the ROC curve.

\section{Results}

Tumor subtypes included 28 adenocarcinomas, 25 mixed adenocarcinomas with bronchioloalveolar features, 2 bronchioloalveolar carcinomas, 15 squamous cell carcinomas, and 7 adenosquamous carcinomas. The average age at diagnosis was 59 (range from 35 to 79). By FISH, 29\% cases were nonamplified, $18 \%$ had low polysomy, $35 \%$ had high polysomy, and $12 \%$ had gene amplification (Table 1 and Figure 1). CISH failed in two cases, FISH failed in one case, and both techniques failed in two cases.

The ranges of CISH signals recorded in the analyzed cases are shown in Table 2 . In the majority of the nonamplified cases, the number of CISH signals per cell ranged from 2 to 5 (Figure 1b); one case had 2-10 signals per cell. This case was an adenosquamous carcinoma, and technical or sampling bias could be the source of discrepancy, with one of the components (glandular or squamous) possibly not examined by FISH. The number of CISH signals per cell ranged from 2 to 7 in the cases with low polysomy (Figure 1d). For all but one of the high polysomy cases, the number of CISH signals per cell ranged from 2 to 15 (Figure 1f). The case contained up to 20 CISH signals per cell; the corresponding FISH assay was complicated by high background. In gene-amplified cases, the number of CISH signals per cell was estimated at 5-30 with at least focal clustering of signals consistent with tandem gene repeats (Figure $1 \mathrm{~h}$ ).

Significant discrepancies between CISH and FISH scores leading to miscategorization as no

Table 1 Distribution of EGFR copy number for each nonsmall cell lung carcinoma subtype by FISH ${ }^{\text {a }}$

\begin{tabular}{|c|c|c|c|c|c|}
\hline \multirow[t]{2}{*}{ Tumor subtype } & \multicolumn{5}{|c|}{ Number of cases } \\
\hline & Total & Nonamplified & Low polysomy & High polysomy & Gene amplified \\
\hline Adenocarcinoma & 28 & 5 & 4 & 16 & 2 \\
\hline $\begin{array}{l}\text { Adenocarcinoma with bronchioloalveolar } \\
\text { features }\end{array}$ & 25 & 7 & 7 & 9 & 3 \\
\hline Bronchioloalveolar carcinoma & 2 & 2 & - & - & - \\
\hline Squamous cell carcinoma & 15 & 7 & 2 & 1 & 2 \\
\hline Adenosquamous carcinoma & 7 & 1 & 1 & 2 & 2 \\
\hline Total & 77 & 22 & 14 & 27 & 9 \\
\hline
\end{tabular}

${ }^{\mathrm{a}} \mathrm{CISH}$ and/or FISH assays failed in five cases (one adenocarcinoma, one adenocarcinoma with bronchioloalveolar features, two squamous cell carcinomas and one adenosquamous carcinoma). 
Table 2 Summary of EGFR CISH signal ranges for a study set of nonsmall cell lung carcinoma $(n=72)$

A: Distribution of the analyzed cases according to the CISH signal range

\begin{tabular}{lrc}
\hline $\begin{array}{l}\text { Range of CISH signal } \\
\text { numbers }\end{array}$ & $\begin{array}{c}\text { Percentage of } \\
\text { cases }\end{array}$ & (Number) \\
\hline$<3$ & 11 & $(8)$ \\
$2-4$ & 19 & $(14)$ \\
$2-5$ & 7 & $(5)$ \\
$2-7$ & 1 & $(1)$ \\
$2-10$ & 1 & $(1)$ \\
$3-5$ & 6 & $(4)$ \\
$3-6$ & 3 & $(2)$ \\
$3-7$ & 3 & $(2)$ \\
$3-8$ & 3 & $(2)$ \\
$4-6$ & 10 & $(7)$ \\
$4-7$ & 3 & $(2)$ \\
$4-9$ & 7 & $(5)$ \\
$5-10$ & 10 & $(7)$ \\
$5-15$ & 1 & $(1)$ \\
$10-15$ & 6 & $(4)$ \\
5 to $>15$ & 1 & $(1)$ \\
$>15$ & 8 & $(6)$
\end{tabular}

B: Interquartile distribution of analyzed cases according to the CISH signal range

\begin{tabular}{lcc}
\hline CISH signal score & $\begin{array}{c}\text { a } \\
\text { Percentage of } \\
\text { Cases }^{\mathrm{b}}\end{array}$ & (Number) \\
\hline 2.8 & 29 & $(22)$ \\
4.6 & 22 & $(16)$ \\
7.1 & 29 & $(21)$ \\
21.2 & 18 & $(13)$ \\
\hline
\end{tabular}

${ }^{\mathrm{a} C a l c u l a t e d}$ as the geometric mean of the CISH signal range loci tabulated in quartiles of the percentage distribution.

${ }^{\mathrm{b}}$ Due to rounding, percentages may not total 100 .

amplification/low polysomy or high polysomy/gene amplified by CISH occurred in five cases (7\%). The CISH score was greater than the FISH score in three cases and less than the FISH score in two cases.

A narrow range of nuclear EGFR signals was detected by CISH for each case in the majority of cases. The ranges were narrowest (4 points or less) in the cases of nonamplified or low polysomy tumors, with 24 of 26 cases (92\%) demonstrating a range of 4 points or less. The ranges were greatest (up to 25 points) in the gene-amplified tumors, in which exact signal counting was complicated by the high number of tandemly positioned signals, as well as by tumor heterogeneity with only a subset of cells displaying amplification in some cases. In nonamplified, low polysomy, and high polysomy tumors, the range of signals most commonly followed a Gaussian distribution, with $50 \%$ of the cells evaluated falling at or above the mean for each range.

The number of signals detected by CISH correlates with the number recorded by FISH (Spearman's $r=0.81, P<0.0001$, Figure 2).

CISH is not a useful test to discriminate between nonamplified and low polysomy categories when

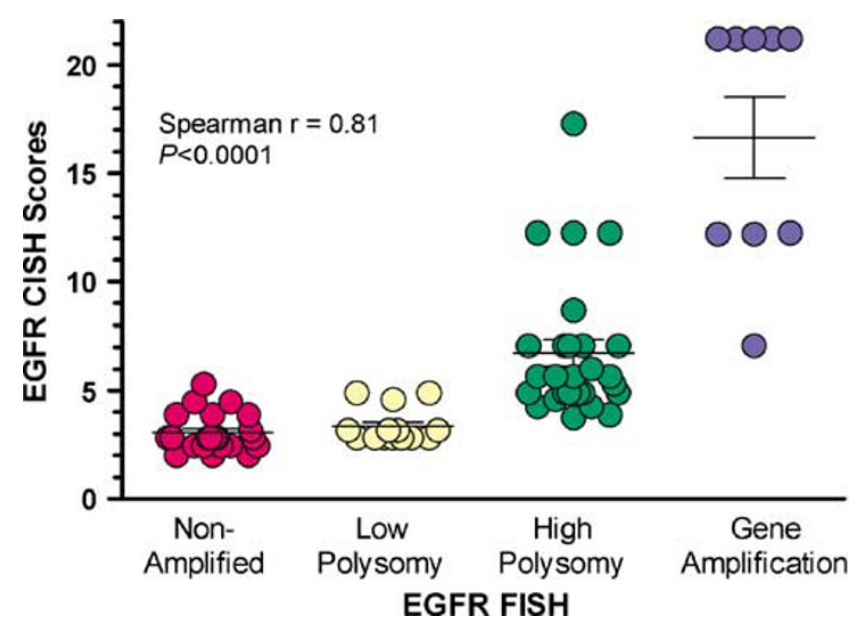

Figure 2 Correlation of the CISH scores with the FISH copy number category by nonparametric analysis (Spearman correlation $r=0.81, P<0.0001)$. Middle lines represent median values with $95 \%$ confidence intervals.

the EGFR signal number is $2.8(P=0.09)$, as the test has a low sensitivity of $50 \%$ and specificity of $68 \%$ (area under the curve $=0.67$, Figure 3a). However, when EGFR signal number is 4.5, CISH is a powerful test with a sensitivity of $89 \%$ and specificity of $89 \%$ (area under the curve $=0.97$, Figure $3 \mathrm{~b}$ ) for discriminating between low polysomy/low EGFR amplification and high polysomy/EGFR amplification $(P<0.0001)$. When EGFR signal number is 7.1, CISH is a powerful test with a sensitivity of $78 \%$ and specificity of $81 \%$ (area under the curve $=0.91$, Figure 3c) for discriminating between high polysomy and EGFR amplification $(P=0.0003)$.

\section{Discussion}

EGFR gene amplification predicts response to tyrosine kinase inhibitor therapy in at least a subset of nonsmall cell lung carcinoma patients. ${ }^{9,13,21}$ EGFR gene amplification is detected in some EGFRmutation-positive patients as well, although its contribution to disease progression and response to therapy in this subgroup is less clear. ${ }^{8}$ In the present study, we evaluated the utility of CISH in detecting EGFR gene copy number by comparing EGFR CISH with FISH results in a cohort of patients known to have a high prevalence of EGFR mutations. ${ }^{1,9}$ We used discriminant analysis to identify CISH cut-off points that differentiate between nonsmall cell lung carcinoma with low and high levels of gene amplification. We demonstrated that CISH can reliably stratify nonsmall cell lung carcinoma according to EGFR gene copy number and propose that CISH serve as a valuable tool for identifying nonsmall cell lung carcinoma with abnormal EGFR gene copy number.

EGFR gene amplification can be assessed by quantitative PCR (qPCR) ${ }^{9}$ or by FISH analysis. ${ }^{8,21,22}$ By these methods, various definitions have been 
a

\begin{tabular}{|r|r|r|}
\hline CISH score & Sensitivity (\%) & Specificity (\%) \\
\hline 2.0 & 100 & 14 \\
\hline 2.5 & 100 & 36 \\
\hline 2.8 & 50 & 68 \\
\hline 3.2 & 21 & 73 \\
\hline 3.9 & 21 & 86 \\
\hline 4.5 & 21 & 95 \\
\hline 4.6 & 14 & 95 \\
\hline 4.9 & 0 & 95 \\
\hline
\end{tabular}

b

\begin{tabular}{|r|r|r|}
\hline CISH score & Sensitivity (\%) & Specificity (\%) \\
\hline 2.0 & 100 & 8 \\
\hline 2.5 & 100 & 22 \\
\hline 2.8 & 100 & 61 \\
\hline 3.2 & 100 & 75 \\
\hline 3.7 & 97 & 75 \\
\hline 3.9 & 94 & 83 \\
\hline 4.2 & 89 & 83 \\
\hline 4.5 & 89 & 89 \\
\hline 4.6 & 86 & 92 \\
\hline 4.9 & 69 & 97 \\
\hline 5.3 & 67 & 100 \\
\hline 5.7 & 56 & 100 \\
\hline 6.0 & 53 & 100 \\
\hline 7.1 & 33 & 100 \\
\hline 8.7 & 28 & 100 \\
\hline 12.3 & 17 & 100 \\
\hline 17.3 & 14 & 100 \\
\hline & & \\
\hline
\end{tabular}
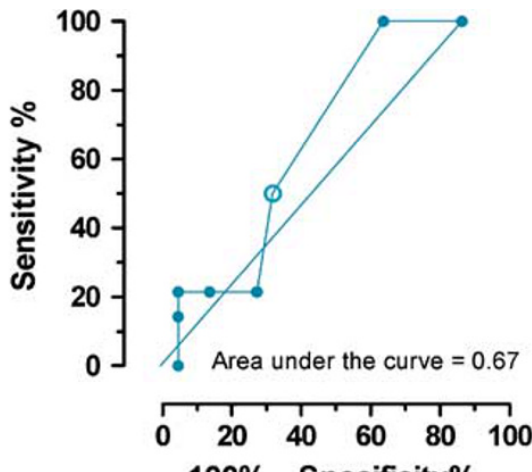

$100 \%$ - Specificity \%
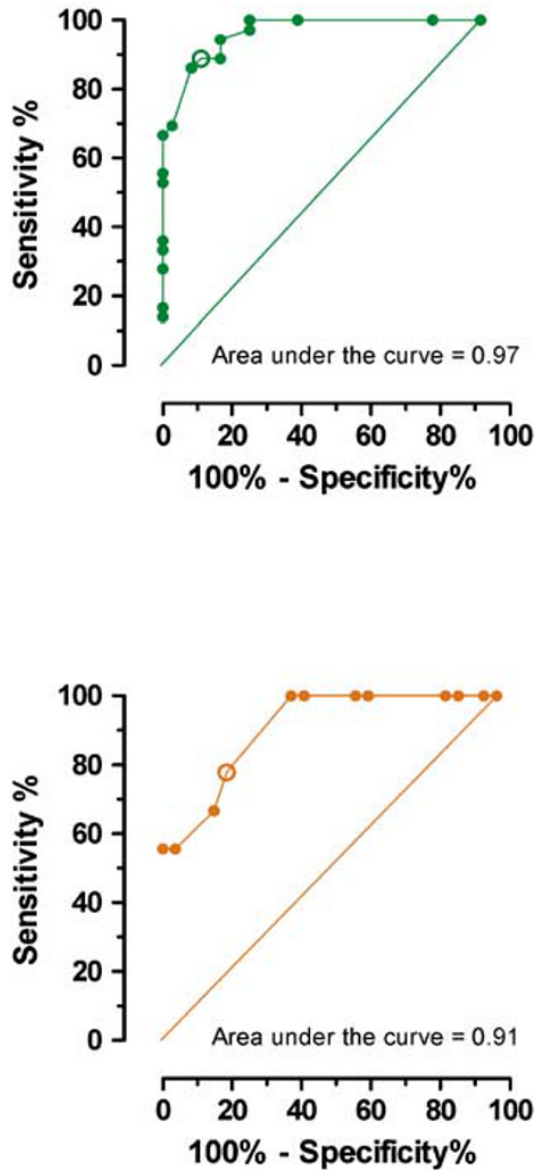

Figure 3 ROC curves derived from logistic regression analysis using CISH signal number to discriminate between FISH categories. (a) No EGFR amplification vs low polysomy. The open circle designates optimal CISH cut-off score of 2.8 with a sensitivity of $50 \%$ and specificity of $68 \%$. (b) No EGFR amplification/low polysomy vs high polysomy/EGFR gene amplification. The open circle designates optimal CISH cut-off score of 4.5 with a sensitivity of $89 \%$ and specificity of $89 \%$. (c) High polysomy vs EGFR gene amplification. The open circle designates optimal CISH cut-off score of 7.1 with a sensitivity of $78 \%$ and specificity of $81 \%$.

described, including 'highly increased copy number' at $\geq 6$ copies by qPCR due to 'selective amplification of mutant alleles'9 or 'gene amplified' at $\geq 15$ copies of EGFR per cell in $>10 \%$ of cells or a ratio of $E G F R$ gene to chromosome of greater than or equal to 2 by FISH. ${ }^{21}$ Like gene amplification, the presence of 3-6 EGFR copies by qPCR, termed a 'moderately increased' copy number, or the presence of $\geq 4$ copies of $E G F R$ in $\geq 40 \%$ of cells by
FISH, termed high polysomy, have been associated with better response to tyrosine kinase inhibitors. ${ }^{8,9,21}$

The practicality of FISH is limited by the need for fluorescence microscopy equipment and technical expertize. CISH provides a simple alternative to FISH; CISH utilizes a chromogen that can be visualized by light microscopy, permitting interpretation while simultaneously examining the tissue 
histology and correlating CISH results with cytoarchitectural details in the examined cancer sections.

In our study, we have demonstrated that the number of signals detected by CISH correlates with the number recorded by FISH (Spearman $r=0.81$, $P<0.0001)$. CISH can effectively discriminate between two previously defined, clinically significant categories of EGFR 'FISH negative' (no amplification and low polysomy) and 'FISH positive' (high polysomy and gene amplified $)^{21}$ nonsmall cell lung carcinoma $(P<0.0001)$ and also between high polysomy and gene-amplified cases $(P=0.0003$, Figure 3). CISH is less effective in discriminating between nonamplified and low polysomy nonsmall cell lung carcinoma cases $(P=0.09$, Figure 3$)$, although the clinical significance of low polysomy in nonsmall cell lung carcinoma is unknown.

There is some consensus in the literature regarding the approach to CISH scoring for HER2 gene amplification in breast cancer. ${ }^{14-16}$ However, there is no such consensus regarding the use of EGFR in lung cancer, and published reports have adopted the HER2 approach, ${ }^{23}$ classifying $3-5$ signals per nucleus as aneuploidy, and $>6$ signals per nucleus in $>50 \%$ of tumor cells as amplification. Our approach to CISH scoring involved evaluating 200 tumor cells per sample, recording the maximum and minimum number of signals seen in each case, and computing the geometric mean to minimize the dispersion effect of wide ranging CISH values. We established cut-off values between no and low polysomy at 2.8 copies per nucleus, between low and high polysomy at 4.5 copies per nucleus and between high polysomy and gene-amplified cases at 7.1 copies per nucleus (Figure 3). CISH is not a useful test in discriminating between nonamplified and low polysomy cases; however, for the latter two comparisons, after rounding to the nearest integer, the adjusted cut-off values are 5 and 7 , respectively. We define nonsmall cell lung carcinoma as: negative for amplification (diploidy and low polysomy) cases with fewer than 5 signals per nucleus; positive for amplification (high polysomy and gene amplification) cases with at least 5 signals per tumor nucleus and/or the presence of large signal clusters.

CISH uses only one probe per slide and therefore a control for polysomy cannot be simultaneously visualized, as it can in FISH. Some authors advocate the use of a centromeric probe on a parallel tissue section to better define the level of polysomy, particularly in borderline cases. ${ }^{15}$ In our study, scoring based only on the EGFR signal number allows for good discrimination between low and high polysomy cases and between high polysomy and gene-amplified cases, which in most cases is easily detected as a tight cluster of signals, reflecting tandem replication at the EGFR gene locus $(P<0.0001$ and $P=0.0003$, respectively, Figure $3 \mathrm{~b}$ and $\mathrm{c}$ ). In the borderline cases, particularly those that appear to have between 4 and 5 signals per tumor nucleus, the parallel examination of centromeric signal may be advised, although this approach has not yet been validated. Alternatively, subsequent FISH analysis of borderline cases may be warranted.

Significant discrepancies between CISH and FISH scores were relatively rare (in $6.5 \%$ of cases). In some cases, higher scores by CISH could be attributed to overlapping nuclei leading to an overestimation of signal number per cell. In two cases, the higher scores by CISH may have been due to technical problems on FISH (loss of tissue, failure to detect signals in both tumor components of adenosquamous carcinomas). Lower scores by CISH were seen in two cases with FISH scores that were borderline between low and high polysomy.

In this subset of nonsmall cell lung carcinoma, gene amplification was detected in adenocarcinomas, adenocarcinomas with bronchioloalveolar features, squamous cell carcinomas, and in adenosquamous carcinomas. Published reports detected nonsmall cell lung carcinoma with high polysomy or gene amplification by FISH in $30 \%$ of patients with advanced bronchioloalveolar carcinomas or adenocarcinomas with bronchioloalveolar features in a US study of a heterogeneous Western population that included smokers, ${ }^{21}$ in which activation of the KRAS pathway may play a significant role in tumorigenesis. ${ }^{24}$ In our study set, high polysomy or gene amplification were seen in $50 \%$ of interpretable cases overall and in $55 \%$ of cases, including adenocarcinomas, adenocarcinomas with bronchioloalveolar features, and bronchioloalveolar carcinomas, high percentages that likely reflect the fact that lung cancers in the selected population (Asian, female, nonsmokers) are more likely to be driven by EGFR hyperactivation. ${ }^{25}$

The technical ease and utility of CISH for detection of EGFR gene amplification in patients with nonsmall cell lung carcinoma suggests that this assay could serve an important role in identifying patients with a high number of EGFR copies for assignment to individualized therapeutic regimens. This assay can be rapidly performed in standard clinical immunohistochemistry laboratories and interpreted by the pathologist under light microscopy. However, EGFR gene copy number analysis should be performed in conjunction with EGFR mutation analysis for selection of EGFR-targeted therapies in patients with lung cancer. As the understanding of mechanisms and importance of EGFR hyperactivation in nonsmall cell lung carcinoma evolves, it will be important to determine the level of polysomy that predicts tumor progression and/or response to tyrosine kinase inhibitors. Our study shows that nonsmall cell lung carcinoma with at least five EGFR gene signals in tumor cell nuclei by CISH have high polysomy or gene amplification by FISH. Based on our findings, additional studies are warranted to determine whether a classification algorithm that includes EGFR copy number by CISH, 
in addition to EGFR mutation analysis, can contribute to stratification of patients with nonsmall cell lung carcinoma for clinical trials and identify a subset that should be treated with tyrosine kinase inhibitors.

\section{Acknowledgement}

This study was supported by CA90578, CA074386, and CA092824 from the National Institutes of Health. Dr Chirieac was supported by W81XWH06-1-0303 from the Department of Defense. We thank Ms Sarah Spill for her editorial assistance in preparing the manuscript and Ms Georgiana Kuhlmann for her technical assistance.

\section{Disclosure/conflict of interest}

There is no duality of interest to be disclosed.

\section{References}

1 Sharma SV, Bell DW, Settleman J, et al. Epidermal growth factor receptor mutations in lung cancer. Nat Rev Cancer 2007;7:169-181.

2 Lynch TJ, Bell DW, Sordella R, et al. Activating mutations in the epidermal growth factor receptor underlying responsiveness of non-small-cell lung cancer to gefitinib. N Engl J Med 2004;350:2129-2139.

3 Testa JR, Siegfried JM. Chromosome abnormalities in human non-small cell lung cancer. Cancer Res 1992;52:2702s-2706s.

4 Ettinger DS, Bepler G, Bueno R, et al. Non-small cell lung cancer clinical practice guidelines in oncology. J Natl Compr Cancer Netw 2006;4:548-582.

5 Shigematsu H, Lin L, Takahashi T, et al. Clinical and biological features associated with epidermal growth factor receptor gene mutations in lung cancers. J Natl Cancer Inst 2005;97:339-346.

6 Yang GC, Yao JL, Feiner HD, et al. Lipid-rich follicular carcinoma of the thyroid in a patient with McCuneAlbright syndrome. Mod Pathol 1999;12:969-973.

7 Paez JG, Janne PA, Lee JC, et al. EGFR mutations in lung cancer: correlation with clinical response to gefitinib therapy. Science 2004;304:1497-1500.

8 Cappuzzo F, Hirsch FR, Rossi E, et al. Epidermal growth factor receptor gene and protein and gefitinib sensitivity in non-small-cell lung cancer. J Natl Cancer Inst 2005;97:643-655.

9 Takano T, Ohe Y, Sakamoto H, et al. Epidermal growth factor receptor gene mutations and increased copy numbers predict gefitinib sensitivity in patients with recurrent non-small-cell lung cancer. J Clin Oncol 2005;23:6829-6837.

10 Nomura M, Shigematsu H, Li L, et al. Polymorphisms, mutations, and amplification of the EGFR gene in nonsmall cell lung cancers. PLoS Med 2007;4:e125.

11 Sone T, Kasahara K, Kimura H, et al. Comparative analysis of epidermal growth factor receptor mutations and gene amplification as predictors of gefitinib efficacy in Japanese patients with nonsmall cell lung cancer. Cancer 2007;109:1836-1844.
12 Marchetti A, Martella C, Felicioni L, et al. EGFR mutations in non-small-cell lung cancer: analysis of a large series of cases and development of a rapid and sensitive method for diagnostic screening with potential implications on pharmacologic treatment. J Clin Oncol 2005;23:857-865.

13 Hirsch FR, Varella-Garcia M, Bunn Jr PA, et al. Molecular predictors of outcome with gefitinib in a phase III placebo-controlled study in advanced non-small-cell lung cancer. J Clin Oncol 2006;24: 5034-5042.

14 Gong Y, Gilcrease M, Sneige N. Reliability of chromogenic in situ hybridization for detecting HER-2 gene status in breast cancer: comparison with fluorescence in situ hybridization and assessment of interobserver reproducibility. Mod Pathol 2005;18: 1015-1021.

15 Hanna WM, Kwok K. Chromogenic in situ hybridization: a viable alternative to fluorescence in situ hybridization in the HER2 testing algorithm. Mod Pathol 2006;19:481-487.

16 Tanner M, Gancberg D, Di Leo A, et al. Chromogenic in situ hybridization: a practical alternative for fluorescence in situ hybridization to detect HER-2/neu oncogene amplification in archival breast cancer samples. Am J Pathol 2000;157:1467-1472.

17 Travis WD, Brambilla E, Muller-Hermelink HK, et al. Pathology and Genetics: Tumours of the Lung, Pleura, Thymus, and Heart. World Health Organization Classification of Tumours. IARC Press: Lyon, 2004.

18 Beasley MB, Brambilla E, Travis WD. The 2004 world health organization classification of lung tumors. Semin Roentgenol 2005;40:90-97.

19 Greene FL PD, Fleming ID, Fritz A. AJCC Cancer Staging Manual, 6th edn. Springer-Verlag: New York, 2002.

20 Thorner PS, Ho M, Chilton-MacNeill S, et al. Use of chromogenic in situ hybridization to identify MYCN gene copy number in neuroblastoma using routine tissue sections. Am J Surg Pathol 2006;30: $635-642$.

21 Hirsch FR, Varella-Garcia M, McCoy J, et al. Increased epidermal growth factor receptor gene copy number detected by fluorescence in situ hybridization associates with increased sensitivity to gefitinib in patients with bronchioloalveolar carcinoma subtypes: a Southwest oncology group study. J Clin Oncol 2005;23:6838-6845.

22 Hirsch FR, Varella-Garcia M, Bunn Jr PA, et al. Epidermal growth factor receptor in non-small-cell lung carcinomas: correlation between gene copy number and protein expression and impact on prognosis. J Clin Oncol 2003;21:3798-3807.

23 Awaya H, Takeshima Y, Furonaka O, et al. Gene amplification and protein expression of EGFR and HER2 by chromogenic in situ hybridisation and immunohistochemistry in atypical adenomatous hyperplasia and adenocarcinoma of the lung. J Clin Pathol 2005;58:1076-1080.

24 Ahrendt SA, Decker PA, Alawi EA, et al. Cigarette smoking is strongly associated with mutation of the K-ras gene in patients with primary adenocarcinoma of the lung. Cancer 2001;92:1525-1530.

25 Yang CH, Shih JY, Chen KC, et al. Survival outcome and predictors of gefitinib antitumor activity in East Asian chemonaive patients with advanced nonsmall cell lung cancer. Cancer 2006;107:1873-1882. 